\title{
Aortic dilatation associates to flow displacement and increased circumferential wall shear stress in patients without aortic stenosis - a prospective clinical study
}

S. Petteri Kauhanen $\mathrm{BM}^{1,2}$, Marja Hedman $\mathrm{MD}, \mathrm{PhD}^{2}$, Elina Kariniemi MD 3 , Pekka Jaakkola MD, $\mathrm{PhD}^{4}$, Ritva Vanninen $\mathrm{MD}, \mathrm{PhD}^{2}$, Petri Saari $\mathrm{MD}, \mathrm{PhD}^{2}$, Timo Liimatainen $\mathrm{PhD}^{2,5,6}$.

1. School of Medicine, Institute of Clinical Medicine, Faculty of Health Sciences, University of Eastern Finland

2. Department of Clinical Radiology, Kuopio University Hospital, Imaging Center, Finland

3. Department of Clinical Physiology and Nuclear Medicine, Kuopio University Hospital, Imaging Center, Finland

4. Department of Heart and Thoracic Surgery, Kuopio University Hospital, Heart Center, Finland

5. Research Unit of Medical Imaging, Physics and Technology, University of Oulu, Oulu, Finland

6. Department of Diagnostic Radiology, Oulu University Hospital, Oulu, Finland

Corresponding author:

S. Petteri Kauhanen, BM

Department of Clinical Radiology, Kuopio University Hospital, Imaging Center, Finland

E-mail: petkau@student.uef.fi _and telephone number: +358445140894

PO Box 100, Puijonlaaksontie 2

70029 KYS, Finland

Grant Support

S. Petteri Kauhanen: Personal grant from the will of Oiva Vaittinen and the Rättimäki

Cardiovascular Society

4D Flow MRI of Aortic Dilatation 


\section{ABSTRACT}

\section{Background}

The relationship between blood flow characteristics and ascending aortic (AA) dilatation has not been studied in patients with a tricuspid aortic valve (TAV) without aortic stenosis.

\section{Purpose}

To evaluate whether four-dimensional (4D) flow characteristics determined in MRI are related to AA dilatation by comparing dilated AA and non-dilated AA subjects with TAV.

\section{Study type}

Prospective.

\section{Population}

20 patients with dilated AA, 20 age-matched patients with non-dilated AA.

\section{Field Strength/Sequence}

1.5T/4D flow, 2D flow and anatomic images.

\section{Assessment}

Altogether 16 different 4D flow parameters were assessed in 10 planes in the thoracic aorta. Intra- and inter-observer reproducibility were analyzed.

\section{Statistical test}

Independent T-test for normal-distributed and Mann-Whitney-test for skewed distributed parameters were used. Paired-samples T-test was used to compare 2D and 4D flow parameters. Intraclass correlation coefficient (ICC) was used in intra- and inter-observer reproducibility analysis.

\section{Results}

Aortic flow was displaced from the center line of the aorta in the proximal and tubular planes. Flow displacement (FD) was greatest in the proximal plane of AA and was higher in dilated AA $(4.5 \%$, range $3.0-5.8 \%)$ than in non-dilated AA $(2.0 \%, 1.0-3.0 \%, \mathrm{p}<0.001)$. Total wall shear stress (WSS) values were 1.3 \pm 0.4 times higher on the displaced side than on the opposite side of the aorta $(\mathrm{p}<0.01)$. The circumferential WSS $\left(\mathrm{WSS}_{\mathrm{C}}\right)$ ratio to total WSS was greater in dilated AA, being $0.48 \pm 0.11$ vs. $0.32 \pm 0.09$ in the inner curvature of the proximal $\mathrm{AA}(\mathrm{p}<0.001)$ and $0.37 \pm 0.11$ vs. $0.26 \pm 0.07$ in whole aortic ring in the distal AA $(\mathrm{p}<0.001)$. Depending on $4 \mathrm{D}$ flow parameter, reproducibility varied from excellent $(\mathrm{ICC}=0.923)$ to very low $(\mathrm{ICC}=0.204)$.

\section{Data conclusion}

The present study demonstrates that 4D flow measurements help to visualize the pathological flow patterns related to aortic dilatation. Flow displacement and an increased WSSc/WSS ratio are significantly associated with AA dilatation.

\section{Key words}

4D flow MRI, ascending aorta, dilatation, flow displacement, wall shear stress, reproducibility 


\section{INTRODUCTION}

Ascending aortic (AA) dilatation is usually asymptomatic until the aorta ruptures (1). The overall mortality rate of a ruptured thoracic aortic dilatation (TAD) is extremely high (97\% - $100 \%$ ) (2). AA dilatation is often diagnosed by incidental imaging which has been performed for some other purpose (1). The incidence of TAD in the American population is approximately 10/100 000 and is similar in both genders $(1,3)$. This dilatation is disproportionately present in certain genetic syndromes such as Marfan (4), Loyes-Diezt (5), Turner (6) and Vascular Ehlers-Danlos (7). However, most patients have a non-syndromic form of TAD with other common etiologies such as atherosclerosis and aortic valve stenosis. A subject is more likely to exhibit TAD if a first degree relative has aortic dilatation; then it is called the familial form of TAD (8). TAD can develop both in the case of a bicuspid (BAV) and a tricuspid aortic valve (TAV) although it is more frequent in the case of BAV (9).

The diagnosis of TAD is currently based on morphometric measures with CT or MR angiography (10). Echocardiography is typically used for follow-up of aortic valvular diseases combined to proximal aortic dilation (10). In the dilated AA, altered blood flow velocities and displaced flow patterns have been reported (11). If it were possible to quantitate these alterations in aortic flow, this might provide valuable information as an adjunct to morphometric determinations. One such approach is four-dimensional (4D) flow MRI, which can define blood flow velocities in three directions at different time points during the cardiac cycle (12).

In patients with BAV, the increases in wall shear stress (WSS) values might explain the higher prevalence of aortic dilatation as compared to patients with TAV $(13,14)$. BAV also causes a helical systolic flow pattern in the AA (15). In patients with a dilated AA and a TAV, total WSS values are lower as compared to those with a non-dilated AA (16-18). Aortic stenosis increases WSS in AA, thus the function of the aortic valve has been shown to have an impact on the development of AA dilatation (18).

The aim of the present study was to detect the 4D flow MRI characteristics that are related to AA dilatation without aortic stenosis.

\section{MATERIALS AND METHODS}

\section{Patient Population}


Local Ethical Committee approved this prospective study. All volunteers and patients were interviewed by the research physician and signed an informed study consent before participation. Patients did not have any contraindications for MRI and no pediatric patients were included. All imaging was performed without contrast agents.

This prospective study included consecutive patients who were scheduled for follow-up due to a dilated AA (diameter $\geq 42 \mathrm{~mm}$ ) between July 2017 and March 2018. After excluding five patients with BAV and three with mechanical aortic valve altogether 20 patients with a dilated AA and with TAV were included. We recruited a control group of 20 healthy volunteers with TAV and without AA dilatation (AA diameter less than $42 \mathrm{~mm}$ ) matched for age, gender and body mass index. No cases with aortic valve stenosis were found in either group. Patient characteristics are listed in table 1.

\section{MRI}

Magnetic resonance imaging (MRI) was performed with a Siemens MAGNETOM Aera, 1.5 $\mathrm{T}$ (Siemens GmbH, Erlangen, Germany). The 4D flow sequence extended the imaging time by approximately 20 minutes depending on the patient's heart rate. The total imaging time for all sequences was approximately 45 minutes.

\section{MR Angiography}

After breath-hold scout imaging, MR angiography without administration of contrast agents was performed to measure the dimensions of the aorta during free-breathing. The procedure was based on true fast imaging with steady state precession (TRUFI) with a respiration navigator (19). The imaging stack was positioned via axial clipping and sagittal clipping on the breath-hold scout images. The imaging parameters were repetition time $286 \mathrm{~ms}$, echo time $1.5 \mathrm{~ms}$, pixel bandwidth $590 \mathrm{~Hz}$, reconstructed in-plane pixel spacing 0.8 x $0.8 \mathrm{~mm}^{2}$, slice thickness $1.5 \mathrm{~mm}$, number of slices 64 and imaging time approximately 5 minutes.

\section{D Phase Contrast}

2D phase contrast (PC) data was acquired over multiple cardiac cycles using electrocardiogram (ECG)-gated CINE imaging. Imaging was performed without contrast media based on our clinical practice. Based on the anatomical scout images, two slices were prospectively placed on the sinotubular and tubular planes. The imaging parameters were: repetition time $20 \mathrm{~ms}$, echo time $2.7 \mathrm{~ms}$, pixel spacing 1.6 x $1.6 \mathrm{~mm}$, slice thickness $5 \mathrm{~mm}$, number of cine frames 50 and velocity encoding (VENC) $150 \mathrm{~cm} / \mathrm{s}$. VENC describes the 
maximum suspected flow velocity, thus it is the most important parameter for successful 2D PC imaging $(11,20)$.

\section{D Flow MRI}

A standard Siemens PC 4D flow sequence was scanned prospectively with ECG-gating and free breathing without contrast media. Respiration related artifacts were decreased by averaging. The center and orientation of the imaging stack for 4D flow was aligned with the TRUFI angio stack. The 4D flow spatial resolution was set to $2.3 \times 2.3 \times 3.0 \mathrm{~mm}^{3}$, repetition time $42 \mathrm{~ms}$, number of segments 2, echo time $2.8 \mathrm{~ms}$, temporal resolution same as TR, flip angle $7^{\circ}, 18-25$ cardiac phases, VENC $150 \mathrm{~cm} / \mathrm{s}$ and 15 slices. The imaging parameters were selected in line with the 4D flow MRI consensus statement (21).

\section{Flow Parameter Analysis}

\section{D Flow Parameters}

A retrospective 2D flow data analysis was conducted using syngo.via ${ }^{\circledR}$ (Version: VB30A, Siemens GmbH, Erlangen, Germany) software. Peak flow, peak velocity and regurgitation fraction were quantified from the sinotubular junction and tubular planes.

\section{D Flow Parameters}

Flow was evaluated in 10 planes (Fig. 1) at different levels of the thoracic aorta. We added two extra planes; 1) sinus valsalva and 2) sinotubular junction while the other planes were chosen according to the 4D flow consensus statement (21) as follows; 3) proximal segment, 4) mid ascending aorta, 5) proximal aortic arch, 6) plane immediately after truncus brachiocephalicus, 7) mid aortic arch, 8) end of the aortic arch, 9) proximal descending thoracic aorta and 10) mid descending aorta (10).

Additional parameters assessed, together with the 2D flow measurements, were cardiac output ( $\mathrm{ml} / \mathrm{min}$ ), forward flow $(\mathrm{ml})$, backward flow $(\mathrm{ml})$, net forward volume (forward minus backward flow, ml), regurgitation fraction (ratio between backward and forward flow, \%), cross-sectional aortic area $\left(\mathrm{cm}^{2}\right)$, flow displacement (FD) determined as the center of gravity of the blood vessel - center of eccentric flow / diameter of vessel (\%) (22). FD was further dichotomized as low (less than $5 \%$ ) or moderate ( $5 \%$ or more). The center of gravity of the vessel was based on the cross-sectional contour of the applicable plane, the center of eccentric flow based on velocities within the cross-sectional contour of the applicable plane and the diameter of the vessel based on the area which is defined by the cross-sectional 
contour of the applicable plane. All 4D flow results were analyzed with CAAS MR 4D flow 2.0 (Pie Medical Imaging, Maastricht, Netherlands) software.

\section{Wall Shear Stress}

Total wall shear stress (WSS), circumferential wall shear stress WSS $_{\mathrm{C}}$, parallel to emitter plane) and axial wall shear stress (WSS $\mathrm{A}$, perpendicular to emitter plane) were calculated based on the following equation

$$
\gamma=\left.\frac{\delta V_{\text {parallel }}(r)}{\delta r}\right|_{r=0}
$$

Where $\gamma$ is wall shear rate, $r$ is the radial distance perpendicular to the vessel wall and $V_{\text {parallel }}$ is blood flow parallel to the vessel wall which contributes to shear against the wall. WSS was calculated by multiplying $\gamma$ with the viscosity of blood which was set to $4 \frac{\mathrm{Ns}}{\mathrm{m}^{2}}$ for all dilated and non-dilated aortas $(23,24)$.

The software measured WSS, $\mathrm{WSS}_{\mathrm{C}}$ and $\mathrm{WSS}_{\mathrm{A}}$ values at $4^{\circ}$ intervals. The aortic ring was divided into six sixty-degree segments. One segment included a mean of 15 measuring points. The starting point $\left(0^{\circ}\right)$ was defined to lie in the inner curve of the AA (Fig. 1). Segment 1 covers the AA wall from $0^{\circ}$ to $60^{\circ}$ in a counter-clockwise direction with the rest of the segments following in sequential order. WSS, WSSc and WSSa were measured from planes one to five covering the whole AA. Maximum WSS values are given separately for every plane of the AA.

\section{Intra- And Interobserver Reproducibility}

Observer A (S.P.K) first performed all measurements of the 40 patients guided by an imaging cardiologist. To assess intra-observer reproducibility, Observer A repeated 4D flow and aortic dimension measurements after five months blinded to the previous measurements. To assess inter-observer reproducibility, Observer B (E.K) independently reconstructed 4D flow slices and plane locations and performed the 4D flow measurements blinded to the results of Observer A. WSS analysis was performed separately for planes 1-5 and for 6 segments in each plane. In addition, the aortic dimensions were re-measured by Observer $\mathrm{C}$ (M.H) in the non-dilated group and by Observer D (P.S) in the dilated group.

\section{Statistical Analysis}


All results are presented as median \pm range when the parameters had a skewed distribution and as mean \pm standard deviation if the parameters were normally distributed. Statistical analysis was performed in collaboration with a biostatistician. The statistical significances of differences in flow parameters between the dilated and the non-dilated AA groups were analyzed using independent samples T-test for normally and Mann-Whitney test for skewed distributed parameters. Associations between two sets of parameters were tested using the Spearman Correlation -test.

Inter-technique reproducibility between the 2D and 4D flow parameters and intra- and interobserver reproducibility of the 4D flow parameters were analyzed by paired samples T-test. Intraclass correlation coefficients (ICC) using a two-way mixed affects model with absolute agreement were used to calculate intra- and inter-observer reproducibility. ICC values from 0.0 to 0.2 were considered negligible, from 0.2 to 0.4 very low, from 0.4 to 0.7 moderate, from 0.7 to 0.9 high, and from 0.9 to 1.0 very strong. In addition, coefficients of variability $(\mathrm{CV} \%)$ were assessed by dividing the SD of the differences by the mean of the parameter under consideration. In the current study, group variability with a CV\% of $<8 \%$ was considered homogenous, from 8 to $12 \%$ average, and $>12 \%$ diverse (25). Cohen's kappa was used to study reproducibility of the dichotomized FD measurements; kappa values from 0.0 to 0.2 were considered slight, from 0.2 to 0.4 fair, from 0.4 to 0.6 moderate, from 0.6 to 0.8 substantial, and from 0.8 to 1.0 near perfect (26).

In order to avoid false positive significances due to the multiple comparisons caused by the high number (258) of variables, a false discovery rate (FDR) method was applied. FDR reduces statistical significances in a small study population. The statistical significance level was set to $\mathrm{p}<0.05$ and high statistical significance to $\mathrm{p}<0.01$. All statistical analyses were carried out with SPSS Statistic 23 -software (IBM, Chicago, USA).

\section{RESULTS}

The baseline characteristics of the study subjects are shown in Table 1. Patients' mean age, height, weight, gender or risk factors for cardiovascular diseases did not differ between the dilated AA and the non-dilated AA groups. All patients had a TAV and none of them had an aortic valve stenosis.

In the dilated AA group, blood flow was displaced most prominently to the lateral side in plane 3 (Fig 2). Median FD was $4.5 \%(3.0-5.8 \%)$ in the dilated AA group and $2.0 \%$ (1.0$3.0 \%)$ in the non-dilated AA group, $\mathrm{p}<0.001$. When FD was dichotomized as low $(<5 \%)$ 
or moderate ( $\geq 5 \%), 50 \%$ of the cases $(n=10 / 20)$ in the dilated group had moderate FD while $5.0 \%$ of the cases $(n=1 / 20)$ in the non-dilated group had moderate FD ( $\mathrm{p}=0.001)$. WSS was higher on the lateral side of the aorta where blood flow was displaced, being 1.3-times higher on the side of the aortic wall that was closest to the center of flow as compared to the opposite side $(\mathrm{p}<0.01)$. FD in plane 4 correlated to the maximum backward velocities on plane 1, Fig. 3.

In the dilated AA group, regurgitation fraction was increased in planes 3-10 as compared to the non-dilated AA group (Table 2). The maximum backward flow velocities were higher in the dilated AA group $(37.1 \pm 13.5 \mathrm{~cm} / \mathrm{s})$ than in the non-dilated AA group $(25.7 \pm 9.2 \mathrm{~cm} / \mathrm{s}$, $\mathrm{p}<0.01)$ in planes $1,5,6$ and 7 .

WSS proved to be more circumferential in dilated AAs in comparison to non-dilated AAs. The fraction between circumferential WSS and total WSS $\left(\mathrm{WSS}_{\mathrm{C}} / \mathrm{WSS}\right.$ ) was larger in the dilated AAs when measured from the inner curvature of AA in planes 1-4 and in the whole aortic ring in plane 5 (Table 3). Total WSS values were lower in dilated AA depending on the plane and segment (Table 3).

When the diagnostic performances of the 2D and 4D flow sequences were compared in plane 4 of all 40 subjects, the following parameters did not differ; peak flow, backward flow and regurgitation fraction (Table 4). Peak velocity values obtained by 2D flow were lower $(81 \pm 18 \mathrm{~cm} / \mathrm{s})$ than those obtained by 4D flow $(90 \pm 17 \mathrm{~cm} / \mathrm{s}, \mathrm{p}<0.001)$. The correlation between FD and peak velocity was significant $(r=0.316, p<0.05)$ in the non-dilated group but not in the dilated group $(\mathrm{r}=0.193, \mathrm{p}>0.05)$.

No systematic errors in the 4D flow parameters were observed between Observers A and B, besides FD value in one plane and maximum backward velocity values in two planes. ICC values varied between very low to excellent (Table 5). For the total WSS values, intraobserver reproducibility varied between very low and very high $(\mathrm{ICC}=0.273-0.923)$ depending on plane and sector while the inter-observer reproducibility varied between very low and high $(\mathrm{ICC}=0.204-0.870)$, Fig $4 \mathrm{~A}$. The regurgitation fraction proved to have high reproducibility, Fig 4B, while the maximum backward velocity had low to moderate reproducibility. Both intra-observer and inter-observer reproducibility of the dichotomized FD values was moderate $(\kappa=0.419)$.

\section{DISCUSSION}


The present study demonstrates that 4D flow measurements may help to visualize pathological flow patterns in a clinical cohort of patients with aortic dilatation. Aortic flow displacement was shown to be larger in patients with AA dilatation as compared to those without dilatation. Wall shear stress values were found to be elevated on the lateral side of aorta where the flow was displaced. The circumferential WSS ratio to total WSS was larger in cases of a dilated AA in both the inner curvature of the proximal AA and in the whole aortic ring at the distal AA while total WSS remained decreased or did not differ between the groups. According to our experience, it is important to divide the aortic ring into small segments in order to be able to identify the topography of the differences in WSS.

Relatively few studies exist that have investigated aortic dilatation using 4D flow techniques. One recent study by van Ooji et al. examined 571 patients with 4D flow (18) while the other study populations have been relatively small. The results of Hope et al. indicated that FD correlated significantly with aortic growth (27). Another study by Burk et al. examining patients with dilated AAs revealed that the incidences of a supra-physiologic-helix and vortex flow were greater than the corresponding values in the non-dilated group. In addition, total WSS values were lower in the dilated AA group (16). Alterations in WSS have also been shown to cause changes in endothelial metabolism, which could further lead to vessel wall stiffness and vessel wall remodeling (28-30).

The results of the present study illustrate how flow is displaced from the centerline of the aorta in the ascending aorta. The normal TAV cusp opens at an approximately $75^{\circ}$ angle and flow is located in the centerline of the AA (28). In aortic valvular stenosis, the cusp opening angle and the orientation of the heart influence the angle at which the jet flow leaves the heart. In this study, aortic valvular stenosis was not present in either group, indicating that the differences in FD between the groups may be attributable to other factors such as the cusp opening or the orientation of the heart. Flow displacements in plane 4 were correlated to maximum backward velocities in the plane of the sinus valsalva which could indicate that a failure in the function of aortic valve causes the flow displacement.

Aortic dilatation develops more frequently in patients with BAV than in those with normal TAV. It has been shown that when the BAV has a configuration of either 180 degrees or 140 degrees, this will lead to different flow patterns which will stress the aortic wall to different extents $(13,29)$. The present study with TAV patients highlights that flow displacement in 
the aorta causes increased WSS to the displaced side; this phenomenon is not restricted to patients with a BAV.

In patients with dilated AAs, the fraction between circumferential and total WSS was greater in the inner curvature of the proximal AA and in the whole ring of the distal aorta when compared to the non-dilated AA group. This indicates that the flow pattern is helical throughout the whole dilated AA. Visual streamline inspection of our data shows that helical flow associates with a dominance of circumferential WSS (Fig. 5) which is in agreement with the published literature (13). In contrast, axial WSS is dominant in normal laminar flow (13).

Total WSS values proved to be lower in the dilated AA group in most segments in planes 24. These results are in good agreement with the published literature on dilated AA in patients with TAV (16-18). A recent study suggested that in patients with dilated AAs, the presence of aortic stenosis increased total WSS values in the TAV patients as compared to patients without aortic stenosis in four aortic regions with the exception of those regions on the outer root and proximal AA (18). In the present study, however, aortic stenosis did not explain the differences in flow displacement. Total WSS increased on the side of flow displacement compared to the opposite wall of the aorta. In addition, larger circumferential WSS values were found in the dilated AA group than in the non-dilated AA group.

There were no differences in peak gradients across the aortic valve or aortic regurgitation between the dilated AA and the non-dilated AA groups. However, the regurgitation fraction was higher in planes 3-10 in the dilated AA group which may be a result of the helical flow pattern. Furthermore, the greater maximum backward velocities in the aortic arch most likely resulted from the helical flow pattern detected visually.

The imaging time required for the 4D flow sequence is relatively long, from 15 to 20 minutes as compared to $2 \mathrm{D}$ flow which requires only a few minutes per slice. However, there are several benefits associated with 4D flow measurements e.g. it allows a retrospective evaluation where one can analyze parameters from any part of the thoracic aorta and change the viewpoint of the slice so that it is always perpendicular to the aortic direction. In addition, pathological flow patterns can be visualized by streamlines or as color coded vectors.

When comparing 4D flow-based 2D flow parameters to traditional 2D sequences, we found good agreement between peak flow, backward flow and regurgitation fraction values, in agreement with the published literature $(30,31)$. Only the peak velocity values were greater 
when measured from the 4D flow data; this most likely resulted from the fact that the flow jet direction was not perpendicular to the 2D flow imaging plane. This is supported by the higher correlation between FD and peak velocity in the non-dilated group as compared to that observed in the dilated group.

Despite the possibilities of retrospective evaluation, the reproducibility of the flow parameters of the present study proved to be variable. Intra-observer reproducibility of the total WSS values varied between low and high when analyzed in each plane and sector separately while the reproducibility increased when moving further off the aortic valve plane. Previously, Van Ooji et al. showed that systolic velocity and WSS from 4D flow MRI are reproducible with low inter-observer variability (32). With our best knowledge, reproducibility of FD values has not been studied based on 4D flow data previously and in the present study the moderate reproducibility of the dichotomous FD values associated with differences in aortic lumen demarcation in axial flow images.

The main limitations of this study are the small number of dilated and non-dilated patients $(20+20)$, the long imaging time of the 4D flow sequence, and the lack of respiration gating in the 4D flow sequence. A follow-up study will be needed to evaluate the temporal evolution of the observed FD and circumferential WSS values and their possible prognostic value regarding the growth rate of the AA dilatation. Thus far, the WSS parameter (Pie Medical image analysis) is recommended to be used only for research purposes. However, clinical studies like the present one can offer information on the feasibility of WSS also in clinical practice.

In conclusion 4D flow measurements may help to visualize the pathological flow patterns related to aortic dilatation also in patients with a tricuspid aortic valve without aortic stenosis. Quantitative measurements may provide detailed information about the topography of the flow displacement and circumferential wall shear stress; however, the measurements require experience and may show interobserver variability. 


\section{REFERENCES}

(1) Elefteriades JA, Sang A, Kuzmik G, Hornick M. Guilt by association: paradigm for detecting a silent killer (thoracic aortic aneurysm). Open Heart 2015 Apr 24;2(1):000169. eCollection 2015.

(2) Johansson G, Markström U, Swedenborg J. Ruptured thoracic aortic aneurysms: a study of incidence and mortality rates. J Vasc Surg 1995 Jun;21(6):985-988.

(3) Dudzinski DM, Isselbacher EM. Diagnosis and Management of Thoracic Aortic Disease. Curr Cardiol Rep 2015 Dec;17(12):106.

(4) Dean JCS. Marfan syndrome: clinical diagnosis and management. Eur J Hum Genet 2007 Jul;15(7):724-733.

(5) Van Laer L, Dietz H, Loeys B. Loeys-Dietz syndrome. Adv Exp Med Biol 2014;802:95-105.

(6) Wong SC, Cheung M, Zacharin M. Aortic dilatation and dissection in Turner syndrome: what we know, what we are unclear about and what we should do in clinical practice? Int J Adolesc Med Health 2014;26(4):469-488.

(7) Hasham SN, Guo D, Milewicz DM. Genetic basis of thoracic aortic aneurysms and dissections. Curr Opin Cardiol 2002 Nov;17(6):677-683.

(8) Milewicz DM, Guo D, Tran-Fadulu V, Lafont AL, Papke CL, Inamoto S, et al. Genetic basis of thoracic aortic aneurysms and dissections: focus on smooth muscle cell contractile dysfunction. Annu Rev Genomics Hum Genet 2008;9:283-302.

(9) Verma S, Siu SC. Aortic dilatation in patients with bicuspid aortic valve. N Engl J Med 2014 May 15,;370(20):1920-1929.

(10) Erbel R, Aboyans V, Boileau C, Bossone E, Bartolomeo RD, Eggebrecht H, et al. 2014 ESC Guidelines on the diagnosis and treatment of aortic diseases: Document covering acute and chronic aortic diseases of the thoracic and abdominal aorta of the adult. The Task Force for the Diagnosis and Treatment of Aortic Diseases of the European Society of Cardiology (ESC). Eur Heart J 2014 Nov 01,;35(41):2873-2926.

(11) Markl M, Schnell S, Wu C, Bollache E, Jarvis K, Barker AJ, et al. Advanced flow MRI: emerging techniques and applications. Clin Radiol 2016 Aug;71(8):779-795.

(12) Dyverfeldt P, Bissell M, Barker AJ, Bolger AF, Carlhäll C, Ebbers T, et al. 4D flow cardiovascular magnetic resonance consensus statement. J Cardiovasc Magn Reson 2015 Aug 10,;17:72.

(13) Meierhofer C, Schneider EP, Lyko C, Hutter A, Martinoff S, Markl M, et al. Wall shear stress and flow patterns in the ascending aorta in patients with bicuspid aortic valves differ significantly from tricuspid aortic valves: a prospective study. Eur Heart J

Cardiovasc Imaging 2013 Aug;14(8):797-804. 
(14) Barker AJ, Markl M, Burk J, Lorenz R, Bock J, Bauer S, et al. Bicuspid aortic valve is associated with altered wall shear stress in the ascending aorta. Circ Cardiovasc Imaging 2012 Jul;5(4):457-466.

(15) Hope MD, Hope TA, Meadows AK, Ordovas KG, Urbania TH, Alley MT, et al. Bicuspid aortic valve: four-dimensional MR evaluation of ascending aortic systolic flow patterns. Radiology 2010 Apr;255(1):53-61.

(16) Burk J, Blanke P, Stankovic Z, Barker A, Russe M, Geiger J, et al. Evaluation of 3D blood flow patterns and wall shear stress in the normal and dilated thoracic aorta using flow-sensitive 4D CMR. J Cardiovasc Magn Reson 2012 Dec 13;14:84.

(17) van Ooij P, Potters WV, Nederveen AJ, Allen BD, Collins J, Carr J, et al. A methodology to detect abnormal relative wall shear stress on the full surface of the thoracic aorta using four-dimensional flow MRI. Magn Reson Med 2015 Mar;73(3):1216-1227.

(18) van Ooij P, Markl M, Collins JD, Carr JC, Rigsby C, Bonow RO, et al. Aortic Valve Stenosis Alters Expression of Regional Aortic Wall Shear Stress: New Insights From a 4Dimensional Flow Magnetic Resonance Imaging Study of 571 Subjects. J Am Heart Assoc 2017 Sep 13,;6(9).

(19) Kunz RP, Oellig F, Krummenauer F, Oberholzer K, Neeb D, Vomweg TW, et al. [Contribution of early systole to total antegrade flow volume in breath-hold phase-contrast flow measurements]. Rofo 2005 May;177(5):637-645.

(20) Stankovic Z, Allen BD, Garcia J, Jarvis KB, Markl M. 4D flow imaging with MRI. Cardiovasc Diagn Ther 2014 Apr;4(2):173-192.

(21) Dyverfeldt P, Bissell M, Barker AJ, Bolger AF, Carlhall CJ, Ebbers T, et al. 4D flow cardiovascular magnetic resonance consensus statement. J Cardiovasc Magn Reson 2015 Aug 10;17:5.

(22) Burris NS, Sigovan M, Knauer HA, Tseng EE, Saloner D, Hope MD. Systolic flow displacement correlates with future ascending aortic growth in patients with bicuspid aortic valves undergoing magnetic resonance surveillance. Invest Radiol 2014 Oct;49(10):635639.

(23) Potters WV, van Ooij P, Marquering H, vanBavel E, Nederveen AJ. Volumetric arterial wall shear stress calculation based on cine phase contrast MRI. J Magn Reson Imaging 2015 Feb;41(2):505-516.

(24) Stalder AF, Russe MF, Frydrychowicz A, Bock J, Hennig J, Mark1 M. Quantitative 2D and 3D phase contrast MRI: optimized analysis of blood flow and vessel wall parameters. Magn Reson Med 2008 Nov;60(5):1218-1231.

(25) Kaur H, Attri R. Morphological and Morphometrical Characterization of Meloidogyne incognita from Different Host Plants in Four Districts of Punjab, India. J Nematol 2013 Jun;45(2):122-127. 
(26) Landis JR, Koch GG. The measurement of observer agreement for categorical data. Biometrics 1977 Mar;33(1):159-174.

(27) Hope MD, Sigovan M, Wrenn SJ, Saloner D, Dyverfeldt P. MRI hemodynamic markers of progressive bicuspid aortic valve-related aortic disease. J Magn Reson Imaging 2014 Jul;40(1):140-145.

(28) Burris NS, Hope MD. 4D flow MRI applications for aortic disease. Magn Reson Imaging Clin N Am 2015 Feb;23(1):15-23.

(29) Roman MJ, Pugh NL, Devereux RB, Eagle KA, Holmes K, LeMaire SA, et al. Aortic Dilatation Associated With Bicuspid Aortic Valve: Relation to Sex, Hemodynamics, and Valve Morphology (the National Heart Lung and Blood Institute-Sponsored National Registry of Genetically Triggered Thoracic Aortic Aneurysms and Cardiovascular Conditions). Am J Cardiol 2017 Oct 01,;120(7):1171-1175.

(30) Bollache E, van Ooij P, Powell A, Carr J, Markl M, Barker AJ. Comparison of 4D flow and 2D velocity-encoded phase contrast MRI sequences for the evaluation of aortic hemodynamics. Int J Cardiovasc Imaging 2016 Oct;32(10):1529-1541.

(31) Gabbour M, Schnell S, Jarvis K, Robinson JD, Markl M, Rigsby CK. 4-D flow magnetic resonance imaging: blood flow quantification compared to 2-D phase-contrast magnetic resonance imaging and Doppler echocardiography. Pediatr Radiol 2015 Jun;45(6):804-813.

(32) van Ooij P, Powell AL, Potters WV, Carr JC, Markl M, Barker AJ. Reproducibility and interobserver variability of systolic blood flow velocity and 3D wall shear stress derived from 4D flow MRI in the healthy aorta. J Magn Reson Imaging 2016 Jan;43(1):236-248. 
Table 1. Baseline characteristics of the patients.

\begin{tabular}{lccc}
\hline \hline & Dilated AA & Non-dilated AA & p-value \\
& $\mathrm{n}=20$ & $\mathrm{n}=20$ & \\
\hline \hline Age (years) & $62.3 \pm 6.0$ & $58.5 \pm 6.3$ & 0.06 \\
Gender (n of male/ female) & $17 / 3$ & $15 / 5$ & 0.7 \\
Height (cm) & $177.6 \pm 7.6$ & $174.7 \pm 6.4$ & 0.2 \\
Weight (kg) & $91.0 \pm 15.0$ & $83.3 \pm 13.2$ & 0.09 \\
BMI (kg/m ${ }^{2}$ ) & $28.8 \pm 4.0$ & $27.3 \pm 4.3$ & 0.9 \\
Diameter of sinus valsalva (mm) & $44.7 \pm 5.1$ & $37.4 \pm 1.7$ & $<0.001$ \\
Diameter of tubular plane (mm) & $43.1 \pm 4.3$ & $35.0 \pm 3.5$ & $<0.001$ \\
Hypertension (n) & 14 & 11 & 0.3 \\
Dyslipidemia (n) & 10 & 5 & 0.1 \\
Smoker (n) & 2 & 4 & 0.4 \\
Diabetes (n) & 4 & 2 & 0.4 \\
Coronary artery disease (n) & 5 & 2 & 0.2 \\
Peak velocity gradient in aortic valve (mmHg) & $6.5 \pm 3.0$ & $6.4 \pm 1.8$ & 0.2 \\
Aortic regurgitation (\%) & $6.5 \pm 8.9$ & $3.6 \pm 1.7$ & 0.9 \\
& & & \\
\hline \hline
\end{tabular}

Note: Values of the continuous variables are mean \pm SD. 
Table 2: Median regurgitation fraction values displayed as percentages and ranges for the dilated and the non-dilated groups. Statistical difference between groups assessed by Mann-Whitney -test.

\begin{tabular}{cccc}
\hline \hline \multicolumn{4}{c}{ Regurgitation Fraction } \\
\hline \hline Plane & Dilated AA n=20 & $\begin{array}{c}\text { Non-dilated AA } \\
\mathrm{n}=20\end{array}$ & $\mathrm{p}$-value \\
\hline 1 & $8.5(1.0-12.3)$ & $3.5(0.3-13.0)$ & 0.461 \\
2 & $1.5(0-10.3)$ & $0(0-2.0)$ & 0.265 \\
3 & $2.0(0.3-8.0)$ & $0(0-0.8)$ & 0.001 \\
4 & $1.0(0-5.8)$ & $0(0-0)$ & 0.002 \\
5 & $1.0(0-3.8)$ & $0(0-0)$ & 0.001 \\
6 & $2.0(0.8-8.5)$ & $0(0-0)$ & 0.001 \\
7 & $5.0(2.8-11.0)$ & $1.0(0-1.0)$ & $<0.001$ \\
8 & $3.0(2.0-8.0)$ & $1.0(0-1.0)$ & 0.003 \\
9 & $2.0(1.0-4.0)$ & $0(0-1.0)$ & 0.001 \\
10 & $1.5(0-5.3)$ & $0(0-0)$ & 0.002 \\
\hline
\end{tabular}

Note: Planes; 1) sinus valsalva, 2) sinotubular junction, 3) proximal segment, 4) mid ascending aorta, 5) proximal aortic arch, 6) plane immediately after truncus brachiocephalicus, 7) mid aortic arch, 8) end of the aortic arch, 9) proximal descending thoracic aorta and 10) mid descending aorta. 
Table 3: Mean values $( \pm \mathrm{SD})$ of circumferential and total wall shear stress ratio $\left(\mathrm{WSS}_{\mathrm{C}} /\right.$ WSS) and total wall shear stress (WSS) in the dilated AA and the non-dilated AA groups.

$\begin{array}{lll}\text { Planes } & \text { Sectors } & \text { WSS }_{\mathrm{C}} / \text { WSS }\end{array}$

\begin{tabular}{cccccccc}
\hline \hline & & Dilated & Non-dilated & p- & Dilated & Non- & p- \\
& & AA & AA & value & AA & dilated AA & value \\
\hline 1 & $6+1$ & $0.43 \pm 0.15$ & $0.26 \pm 0.08$ & $<0.001$ & $851 \pm 377$ & $877 \pm 236$ & 0.8 \\
2 & $5+6+1+2$ & $0.39 \pm 0.12$ & $0.24 \pm 0.08$ & $<0.001$ & $1054 \pm 302$ & $1290 \pm 244$ & 0.01 \\
3 & $6+1+2$ & $0.51 \pm 0.18$ & $0.33 \pm 0.14$ & 0.001 & $711 \pm 187$ & $1026 \pm 234$ & $<0.001$ \\
4 & $6+1$ & $0.59 \pm 0.17$ & $0.45 \pm 0.20$ & 0.017 & $453 \pm 151$ & $659 \pm 203$ & 0.001 \\
5 & All & $0.37 \pm 0.11$ & $0.26 \pm 0.07$ & $<0.001$ & $562 \pm 188$ & $655 \pm 132$ & 0.08 \\
\hline \hline
\end{tabular}

Planes; 1) sinus valsalva, 2) sinotubular junction, 3) proximal segment, 4) mid ascending aorta, 5) proximal aortic arch 
Table 4. Intertechnique comparison of $2 \mathrm{D}$ flow values $(\mathrm{ml} / \mathrm{s})$ obtained from the $2 \mathrm{D}$ and $4 \mathrm{D}$ flow sequences in all subjects in plane 4 (mid ascending aorta). Comparisons were performed with paired-samples T-test.

\begin{tabular}{lccc}
\hline \hline & \multicolumn{2}{c}{ Paired-Samples T-Test } \\
\hline \hline & 4D flow sequence & 2D flow sequence & p-value \\
\hline Peak Flow (ml/s) & $434 \pm 73$ & $436 \pm 90$ & 0.8 \\
Backward Flow (ml) & $4 \pm 15$ & $7 \pm 23$ & 0.5 \\
Regurgitation Fraction (\%) & $7 \pm 30$ & $4 \pm 4$ & 0.5 \\
Peak Velocity (cm/s) & $90 \pm 17$ & $81 \pm 18$ & $<0.001$ \\
\hline
\end{tabular}


Table 5. Intra- and inter-observer reproducibility analysis of the aortic dimensions and 4D flow parameters.

\begin{tabular}{|c|c|c|c|c|c|c|}
\hline & \multirow[b]{2}{*}{ Plane } & \multirow[b]{2}{*}{ Sector } & \multicolumn{2}{|c|}{ Intra-observer } & \multicolumn{2}{|c|}{ Inter-observer } \\
\hline & & & ICC & $\mathrm{CV} \%$ & ICC & CV\% \\
\hline \multirow[t]{35}{*}{ Total WSS } & 1 & 1 & 0.416 & 17.0 & 0.417 & 24.2 \\
\hline & & 2 & 0.671 & 13.6 & 0.416 & 18.6 \\
\hline & & 3 & 0.695 & 13.2 & 0.285 & 20.1 \\
\hline & & 4 & 0.437 & 17.2 & 0.224 & 20.6 \\
\hline & & 5 & 0.357 & 28.4 & 0.251 & 32.1 \\
\hline & & 6 & 0.273 & 30.4 & 0.315 & 34.4 \\
\hline & & All & 0.478 & 20.0 & 0.318 & 25.0 \\
\hline & 2 & 1 & 0.710 & 12.5 & 0.470 & 23.6 \\
\hline & & 2 & 0.758 & 13.0 & 0.443 & 23.7 \\
\hline & & 3 & 0.787 & 10.0 & 0.260 & 24.9 \\
\hline & & 4 & 0.706 & 10.9 & 0.265 & 23.1 \\
\hline & & 5 & 0.779 & 11.5 & 0.231 & 22.3 \\
\hline & & 6 & 0.710 & 11.4 & 0.307 & 24.9 \\
\hline & & All & 0.742 & 11.6 & 0.329 & 23.8 \\
\hline & 3 & 1 & 0.479 & 18.1 & 0.568 & 20.9 \\
\hline & & 2 & 0.551 & 14.4 & 0.485 & 26.1 \\
\hline & & 3 & 0.655 & 17.5 & 0.204 & 29.3 \\
\hline & & 4 & 0.655 & 18.7 & 0.424 & 28.8 \\
\hline & & 5 & 0.686 & 15.4 & 0.585 & 23.5 \\
\hline & & 6 & 0.688 & 16.2 & 0.466 & 22.9 \\
\hline & & All & 0.619 & 16.7 & 0.455 & 25.3 \\
\hline & 4 & 1 & 0.654 & 14.4 & 0.622 & 16.4 \\
\hline & & 2 & 0.770 & 9.5 & 0.621 & 14.2 \\
\hline & & 3 & 0.679 & 10.6 & 0.637 & 14.6 \\
\hline & & 4 & 0.823 & 9.3 & 0.659 & 15.7 \\
\hline & & 5 & 0.731 & 14.1 & 0.611 & 15.6 \\
\hline & & 6 & 0.596 & 16.3 & 0.572 & 19.6 \\
\hline & & All & 0.709 & 12.4 & 0.620 & 16.0 \\
\hline & 5 & 1 & 0.798 & 7.9 & 0.856 & 7.6 \\
\hline & & 2 & 0.873 & 9.6 & 0.846 & 8.9 \\
\hline & & 3 & 0.923 & 6.9 & 0.884 & 8.1 \\
\hline & & 4 & 0.879 & 9.1 & 0.828 & 9.6 \\
\hline & & 5 & 0.832 & 8.6 & 0.669 & 10.3 \\
\hline & & 6 & 0.903 & 5.6 & 0.870 & 7.1 \\
\hline & & All & 0.868 & 8.0 & 0.826 & 8.6 \\
\hline \multirow[t]{10}{*}{$\mathrm{WSS}_{\mathrm{C}} / \mathrm{WSS}$} & 1 & Medial $(6+1)$ & 0.502 & 26.4 & 0.436 & 26.2 \\
\hline & & All & 0.504 & 15.8 & 0.285 & 21.6 \\
\hline & 2 & Medial $(6+1)$ & 0.781 & 19.5 & 0.447 & 27.2 \\
\hline & & All & 0.713 & 13.2 & 0.351 & 21.2 \\
\hline & 3 & Medial $(6+1)$ & 0.586 & 21.3 & 0.491 & 23.9 \\
\hline & & All & 0.594 & 14.6 & 0.362 & 24.2 \\
\hline & 4 & Medial $(6+1)$ & 0.540 & 21.2 & 0.314 & 23.6 \\
\hline & & All & 0.628 & 15.0 & 0.308 & 21.4 \\
\hline & 5 & Medial $(1+6)$ & 0.549 & 19.2 & 0.501 & 21.9 \\
\hline & & All & 0.757 & 12.7 & 0.494 & 22.1 \\
\hline Regurgitation fraction & All & - & 0.885 & 9.7 & 0.829 & 10.3 \\
\hline Maximum backward velocity & All & - & 0.426 & 12.7 & 0.323 & 16.7 \\
\hline Aortic dimensions & All & - & 0.610 & 5.2 & 0.917 & 2.5 \\
\hline
\end{tabular}


ICC $=$ intraclass correlation coefficient. ICC values from 0.0 to 0.2 were considered negligible, from 0.2 to 0.4 very low, from 0.4 to 0.7 moderate, from 0.7 to 0.9 high, and from 0.9 to 1.0 very strong. $\mathrm{CV} \%<8 \%$ was considered homogenous, from 8 to $10 \%$ average, and $>12 \%$ diverse.

Planes; 1) sinus valsalva, 2) sinotubular junction, 3) proximal segment, 4) mid ascending aorta, 5) proximal aortic arch, 6) plane immediately after truncus brachiocephalicus, 7) mid aortic arch, 8) end of the aortic arch, 9) proximal descending thoracic aorta and 10) mid descending aorta. 
A

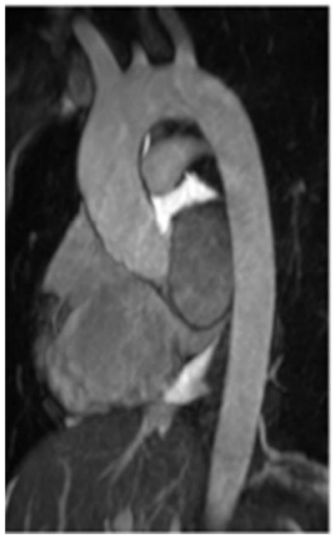

B

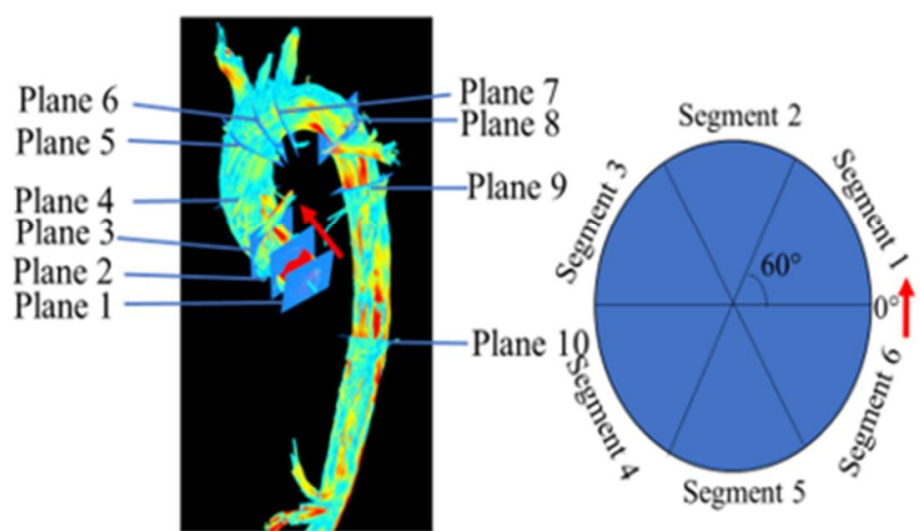

Figure 1. A) Anatomical view of a non-dilated AA in a 23-year-old man by SSFP imaging. The diameter of the sinus valsalva is $37 \mathrm{~mm}$ and the diameter of the tubular plane is $30 \mathrm{~mm}$. B) An illustration of the 4D flow pattern in a non-dilated aorta. The streamline blood jet flow pattern is normal (laminar). The positions of the ten measurement planes are shown. The red arrow is located in the inner curvature of the aorta indicating the zero-point of the WSS measurements. (C) An illustration of the division of the aortic circle into the 6 segments used in these analyses. 


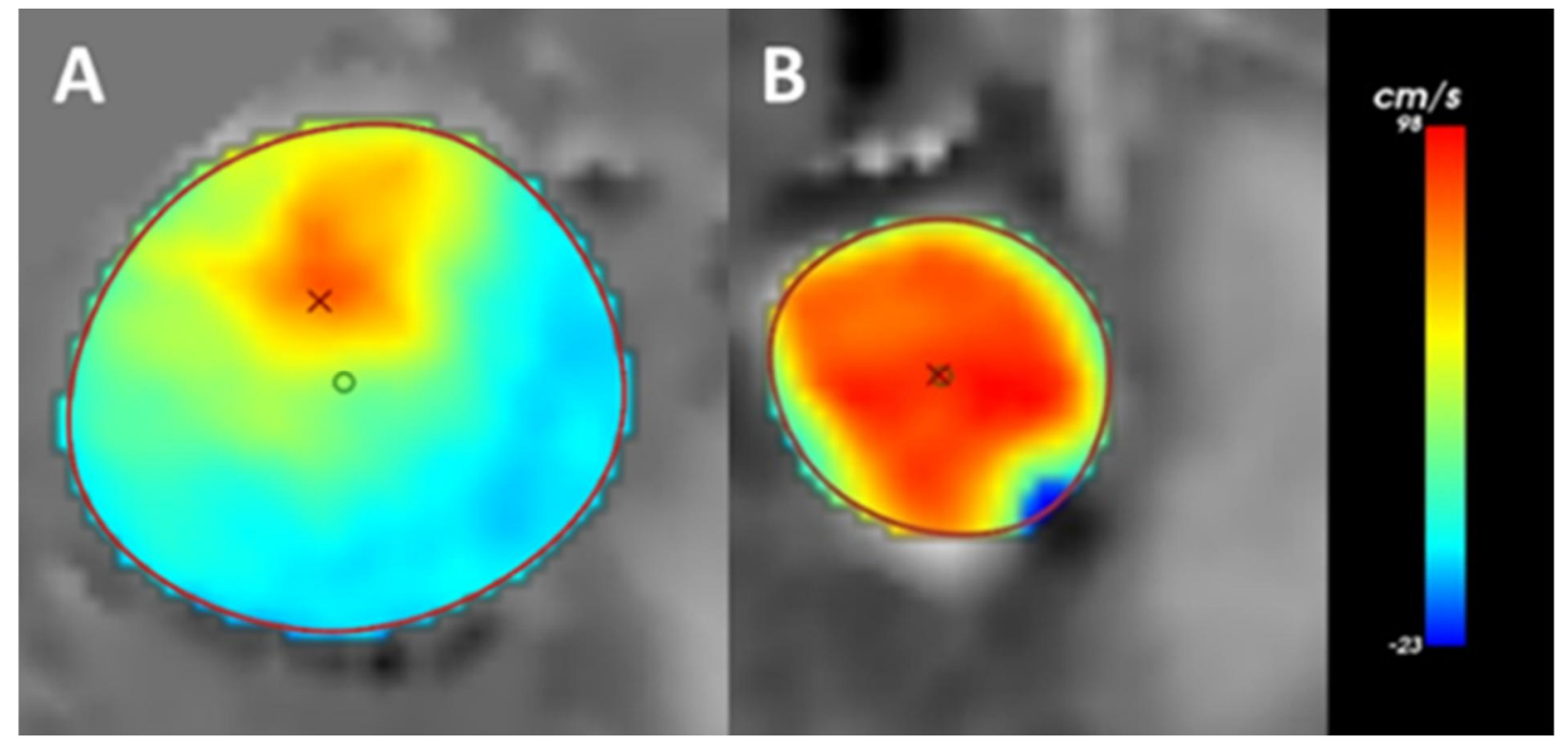

Figure 2. Illustrative images of flow displacement map from plane 3. Circle indicates middle point of aorta and cross indicates center point of flow jet. Flow velocity is coded with colors. A) represents dilated ascending aorta where flow displacement was measured to be $17.2 \%$ and B) represents non-dilated ascending aorta where flow displacement was $2.0 \%$. 


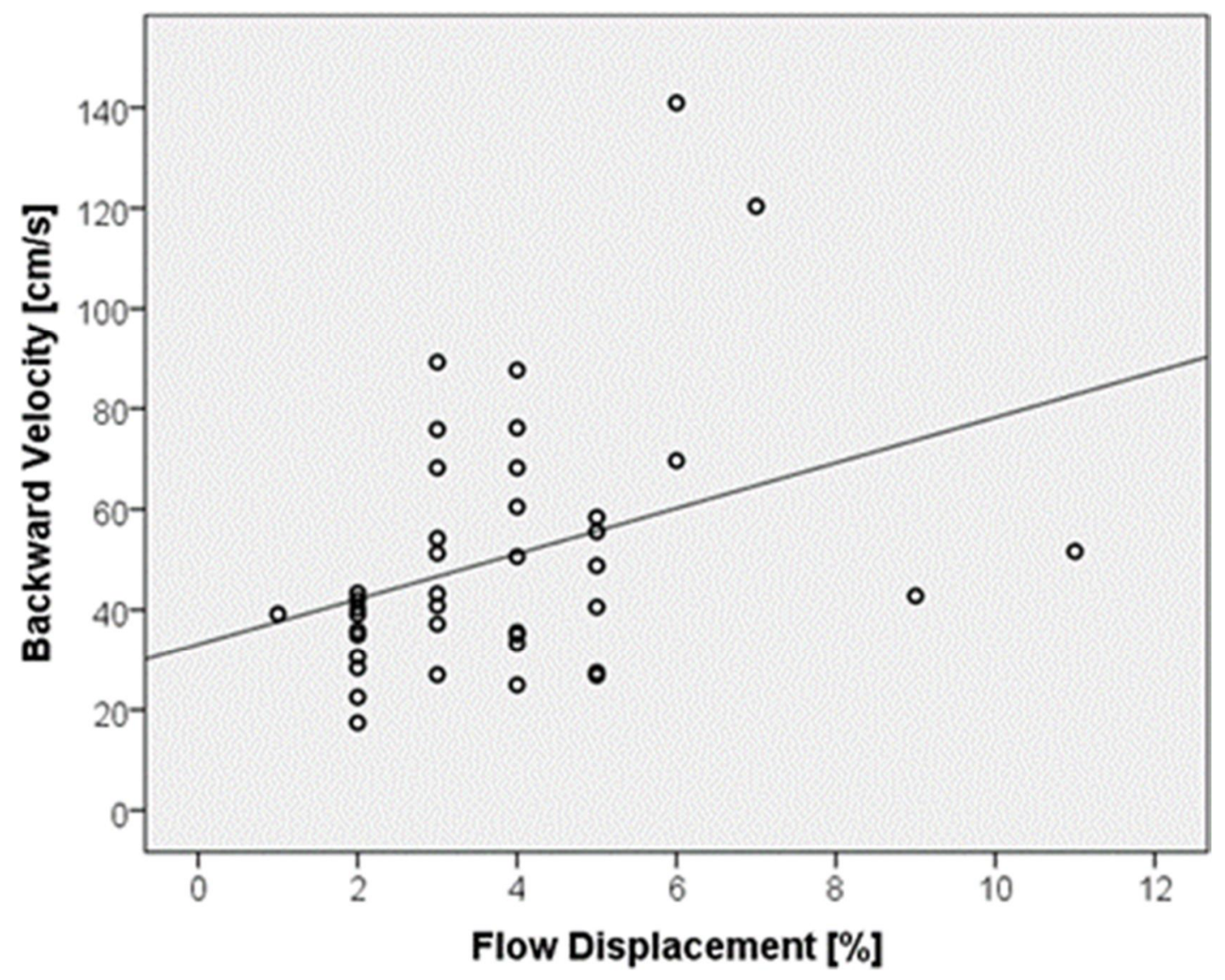

Figure 3. Correlation between flow displacement values (\%) in plane 4 and maximum backward velocity $(\mathrm{cm} / \mathrm{s})$ in the sinus valsalva plane in all subjects $(\mathrm{n}=40, \mathrm{r}=0.397, \mathrm{p}<0.05)$. 

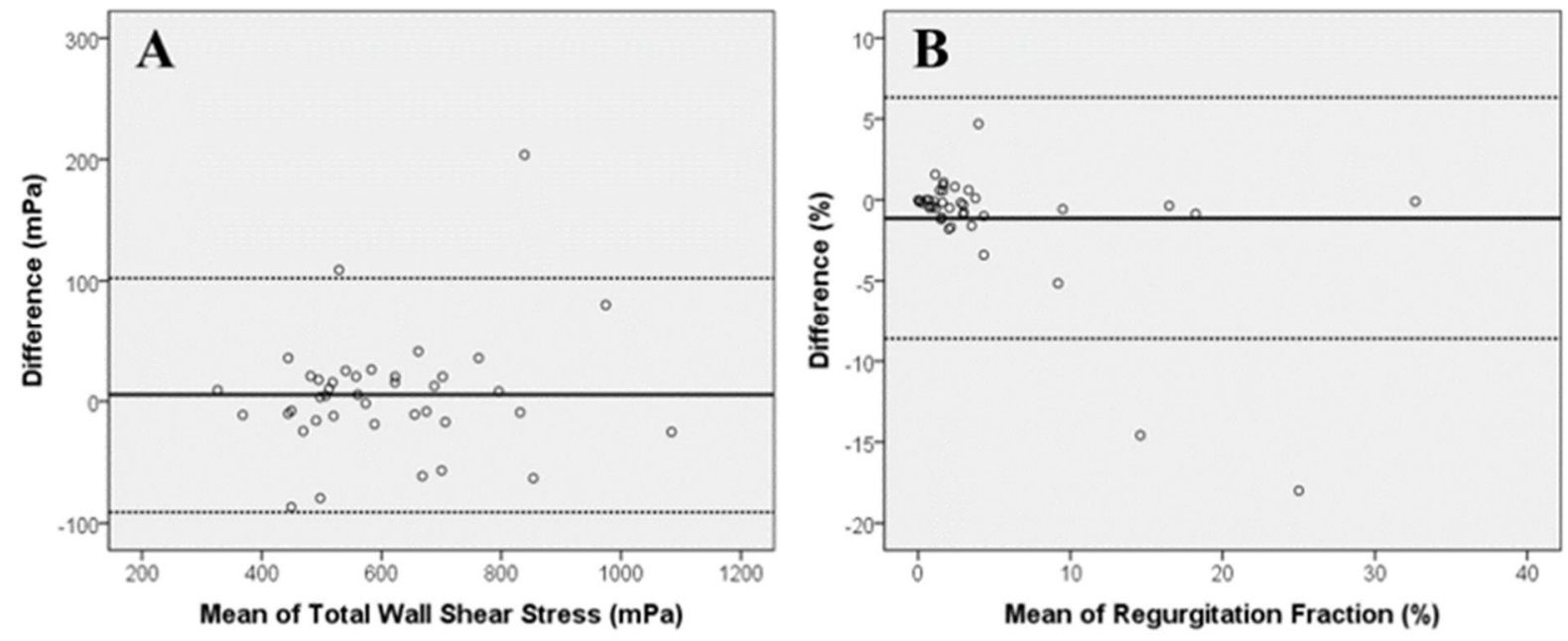

Figure 4. Bland-Altman plot show the inter-observer reproducibility of all 40 patients. The $\mathrm{X}$-axis shows the mean of two parameter measurements and the $\mathrm{y}$-axis shows a difference between respective measurements. The middle line corresponds to the mean difference and the outer dash lines correspond to the $95 \%$ confidence interval. A) represents the total wall shear stress parameter in plane 5 in all sectors. B) represents the regurgitation fraction parameter in all 10 planes. 


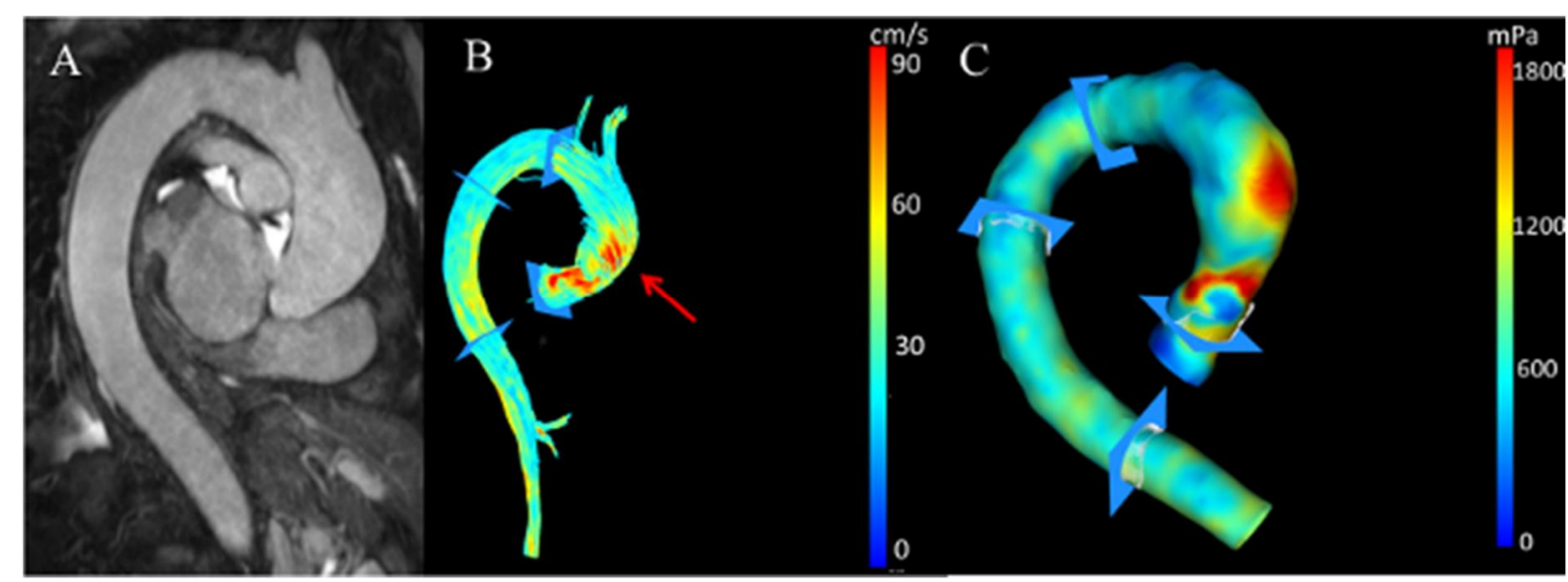

Figure 5. A) An anatomical MR image from a 71-year-old woman with a normal tricuspid aortic valve and AA dilatation. The diameter of the sinus valsalva is $41 \mathrm{~mm}$ and the diameter of the tubular plane is $44 \mathrm{~mm}$. B) 4D flow MR imaging of the dilated AA. Red color indicates areas of increased flow. The blood flow pattern is helical, and its velocity is increased, especially in the outer curvature of the AA (red arrow). C) An image of wall shear stress (WSS) measurements. Total WSS is increased in the outer curvature of the AA (red area) in the same location where the velocity is increased (B). 